\title{
Incidence and factors associated Post-transplantation anemia in kidney transplant patients at a tertiary-care hospital in Thailand
}

\author{
Sayamon Sukkha ${ }^{1}$, \\ Panupong Tiansuwan ${ }^{1}$, \\ Kunvadee Choochaeam ${ }^{2}$, \\ Atiporn Ingsathit $3,4,5$, \\ Punlop Wiwattanathum ${ }^{4,5}$, \\ Jennis Meanwatthana ${ }^{*}$ \\ ${ }^{1}$ Department of Pharmacy, Faculty of \\ Pharmacy, Mahidol University, Bangkok, \\ Thailand \\ 2Pharmacy Division, Faculty of Medicine, \\ Ramathibodi Hospital, Bangkok, Thailand \\ ${ }^{3}$ Section for Clinical Epidemiology and \\ Biostatistics, Faculty of Medicine, \\ Ramathibodi Hospital, Bangkok, Thailand \\ ${ }^{4}$ Department of Medicine, Faculty of \\ Medicine, Ramathibodi Hospital, \\ Bangkok, Thailand \\ ${ }^{5}$ Excellent Center of Organ \\ Transplantation, Ramathibodi Hospital, \\ Mahidol University, Bangkok, Thailand
}

*Corresponding author: Jennis Meanwatthana Jennies.mea@mahidol.edu

\section{KEYWORDS:}

Anemia; Factor; Incidence; Kidney; Transplantation

https://www.pharmacy.mahidol.ac.th/journal/ (C) Faculty of Pharmacy, Mahidol University (Thailand) 2020

\begin{abstract}
The scarce of standard recommendation for posttransplantation anemia (PTA) has resulted in different anemia approaches among transplant centers. The information of PTA in Thai kidney transplant patients is also lacking. We aimed to study the incidence of PTA during one year after transplantation. Factors associated PTA were investigated in Thai population. We performed a retrospective, cohort study from kidney transplant patients between 2014-2015. The primary objective was the incidence of PTA at one year after transplantation. Pre-defined factors were examined to predict the probability of PTA. Of 259 patients, the PTA incidence at one year after transplantation was $43.6 \%$. Factors that increased risk of anemia included urinary tract infection, UTI (OR 2.170, 95\% CI 1.434- 3.248, $P=0.001$ ), ganciclovir or valganciclovir (OR 1.522, 95\% CI 0.926-2.501, $P=$ 0.097 ), history of blood transfusion (OR 1.483 , 95\% CI 1.1771.868, $P=0.001$ ), history of using recombinant human erythropoietin, rHuEpo (OR 1.381, 95\% CI 1.098-1.737, $P=$ 0.006), duration of ESRD (OR 1.032, 95\% CI 1.006-1.059, $P=$ 0.015), and recipient's age (OR 1.019, 95\% CI 1.009-1.030, $P=$ 0.001). PTA was a common complication (43.6\% ) after transplantation in Thai kidney transplant patients. UTI, ganciclovir or valganciclovir, history of blood transfusion, history of using rHuEpo, duration of ESRD as well as recipient's age are associated with an increased risk of PTA. This information can assist practitioners to recognize the tendency of anemia and promising factors to proper management in our transplant center.
\end{abstract}

\section{INTRODUCTION}

Anemia has been a major hematologic complication in all spectrum of kidney diseases. The deficiency in erythropoietin (EPO) hormone is evidently a crucial contributor of anemia in chronic kidney disease (CKD) ${ }^{1}$. The optimal hemoglobin $(\mathrm{Hb})$ target as well as anemia treatment in CKD patients have been extensively provided in various guidelines ${ }^{2-4}$. However, anemia treatment in kidney transplant patients, refers to posttransplantation anemia (PTA), might be under- recognized since there is no current recommendation in those patients. Therefore, 
PTA information particularly in specific population is needed to acknowledge an impact of anemia and to design a proper management associated factors for individual transplant patient.

As kidney allograft does not completely function at the early period after transplantation, serum EPO deficiency has been proposed as a factor related PTA incidence. Winkelmayer WC, et al. showed the relationship between serum EPO level, creatinine level, hematocrit level and the day after transplantation ${ }^{1}$. After immediate post kidney operation, serum EPO has risen up to ninefold from the baseline as a result of blood loss and systemic hypoxia from kidney operation leading to EPO releasing. The second peak of serum EPO level is from kidney allograft performance returns to be well-functioning resulting in three-fold EPO synthesis. After the early phase, serum EPO level will likely become to the normal level if patients have a complete graft function ${ }^{1}$. However, there have been other factors associated PTA besides renal function which also play an important contributor in transplant patients.

The lack of universal standard for the definition and onset of PTA appears to make difficulty for clinical judgment to our patients despite using previous data. Therefore, we aimed to investigate PTA incidence in our Center of Organ Transplantation, Ramathibodi Hospital. PTA was defined by using WHO definition for anemia $^{5}$. The persistence of anemia at 12 months was considered a time point for our investigation. Factors associated PTA were tested to identify the possible factors specifically in Thai kidney transplant patients.

\section{MATERIALS AND METHODS}

This study was a retrospective, cohort study. Eligible patients received new kidney transplantation between 2014- 2015 at our institution. The study was approved by the Ethical Clearance Committee on Human Rights Related to Research Involving Human Subjects, Faculty of Medicine, Ramathibodi Hospital, Mahidol University (MURA 2017/606).

\subsection{Patient selection and the follow-up period}

Patients aged more than 18 years at the day of transplantation were included in the study. Demographic data, clinical and biochemical parameters were collected at baseline. All patients were followed up in outpatient clinic with routine monitoring. One- year follow- up period was investigated in our study. PTA was defined by $\mathrm{Hb}$ less than $13.5 \mathrm{~g} / \mathrm{dL}$ in adult males and less than $12.0 \mathrm{~g} / \mathrm{dL}$ in adult females ${ }^{5}$. We excluded patients who died or lost graft within the follow- up period. All post- transplant records including laboratory parameters, complications, medication used after transplantation were collected at the day of kidney transplantation (day 0), day 3, 7, 14, and month 1, 2, 3, 6, 9, 12 after transplantation.

\subsection{Sample size calculation}

The sample size was estimated based on the following equation ${ }^{6}$ :

$$
\mathrm{N}=\left[\mathrm{Z}^{2}{ }_{\alpha / 2} \times \mathrm{P}(1-\mathrm{P})\right] / \mathrm{M}^{2}
$$

We used $Z$ statistic $(Z)$ for a level of confidence of $95 \%$ with the margin of error $(M)$ of 0.05 . We used the expected prevalence of PTA at 1 year $(\mathrm{P})$ of 0.2 from Imoagene-Oyedeji AE, et $\mathrm{al}^{7}$. The estimated sample size was at least 246 subjects.

\subsection{Outcome measurements}

The primary outcome was the incidence of PTA at one year after transplantation. We also reported the frequency of anemia in various phases during one year period. The phases of PTA were divided into 3 categories ${ }^{8}$ : 1) immediate phase (0-2 weeks), 2) early phase (13 months), and 3) late phase (>3 months-1 year).

The secondary outcome was factors associated PTA. We used twenty-five pre-defined factors as reported in the previous studies to evaluate the relationship of these factors and PTA incidence $^{9-10}$. Pre-defined factors were divided into 1) recipient factors included duration of end stage renal disease (ESRD), estimated glomerular filtration rate (eGFR) at baseline, $\mathrm{Hb}$ at baseline, history of blood loss, history of blood transfusion, history of using rHuEpo, and recipient's age, 2) donor factors included cold ischemic time (CIT), donor's age, expanded criteria donor (ECD), living donor, and standard criteria donor (SCD) and 3) transplant- related factors included angiotensin converting enzyme inhibitors (ACEI) or angiotensin receptor blockers (ARBs), cyclosporin, duration after transplantation, eGFR after transplantation, ganciclovir or valganciclovir, graft dysfunction, mycophenolic acid, serum creatinine (Scr) after 
Table 1 Baseline characteristics of kidney transplant patients

\begin{tabular}{|c|c|c|c|}
\hline Baseline characteristics & 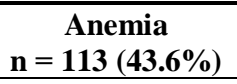 & $\begin{array}{c}\text { No anemia } \\
\mathrm{n}=146(56.4 \%)\end{array}$ & $P$-value \\
\hline \multicolumn{4}{|l|}{ Recipient information } \\
\hline Age, mean $\pm \mathrm{SD}$, year & $45.9 \pm 11.4$ & $43.0 \pm 12.7$ & 0.06 \\
\hline Male, $\mathrm{n}(\%)$ & $37(32.7)$ & $118(80.8)$ & $0.01 *$ \\
\hline $\mathrm{Hb}$, mean $\pm \mathrm{SD}, \mathrm{g} / \mathrm{dL}$ & $10.0 \pm 1.8$ & $11.0 \pm 1.7$ & 0.14 \\
\hline $\mathrm{eGFR}$, mean $\pm \mathrm{SD}, \mathrm{mL} / \mathrm{min} / 1.73 \mathrm{~m}^{2}$ & $8.7 \pm 5.1$ & $7.9 \pm 4.8$ & 0.20 \\
\hline Duration of ESRD, mean $\pm \mathrm{SD}$, year & $6.0 \pm 4.9$ & $5.5 \pm 5.1$ & 0.28 \\
\hline History of blood transfusion, n (\%) & $56(49.6)$ & $63(43.2)$ & 0.26 \\
\hline History of using rHuEpo, n (\%) & $45(39.8)$ & $61(41.8)$ & 0.28 \\
\hline Cold ischemic time, mean $\pm \mathrm{SD}$, hour & $11.5 \pm 9.6$ & $8.2 \pm 9.8$ & $0.05^{*}$ \\
\hline \multicolumn{4}{|l|}{ Induction therapy } \\
\hline -Anti-thymoglobulin, n (\%) & $26(23.0)$ & $18(12.3)$ & $0.03 *$ \\
\hline -Basiliximab, n (\%) & $49(43.4)$ & $57(39.0)$ & 0.49 \\
\hline -No induction, $\mathrm{n}(\%)$ & $38(33.6)$ & $69(47.3)$ & $0.03 *$ \\
\hline \multicolumn{4}{|l|}{ Maintenance therapy } \\
\hline -Tacrolimus, $\mathrm{n}(\%)$ & 79 (69.9) & $92(63.0)$ & 0.25 \\
\hline -Cyclosporin, n (\%) & $35(31.0)$ & $55(37.7)$ & 0.26 \\
\hline $\begin{array}{l}\text {-Mycophenolate mofetil/enteric coated } \\
\text { mycophenolate sodium, } \mathrm{n}(\%)\end{array}$ & $105(92.9)$ & $136(93.2)$ & 0.94 \\
\hline \multicolumn{4}{|l|}{ Donor information } \\
\hline -Living donor (LD), n (\%) & $43(38.1)$ & $85(58.2)$ & $0.01 *$ \\
\hline -Standard criteria donor (SCD), n (\%) & $43(38.1)$ & $44(30.1)$ & 0.18 \\
\hline -Expanded criteria donor (ECD), n (\%) & $27(23.9)$ & $17(11.6)$ & $0.01 *$ \\
\hline
\end{tabular}

eGFR = estimated glomerular filtration rate, $\mathrm{ESRD}=$ end stage renal disease, $\mathrm{Hb}=$ hemoglobin, $\mathrm{rHuEpo}=$ recombinant human erythropoietin; $P$-value of less than 0.05 was considered statistically significant

transplantation, tacrolimus, trimethroprim/sulfamethoxazole, urinary tract infection (UTI), viremia, and viruria.

\subsection{Statistical analysis}

Continuous data was presented as mean \pm SD or median and range. Dichotomous data was presented as rate and percentages. The independent sample t-test for parametric test and Mann-Whitney U test for non-parametric test were used to compare continuous data. Chi-square test was used to compare categorical data. The SPSS Statistics 21.0 for Windows (IBM Corp., Armonk, $\mathrm{NY}$ ) was used and a $P$-value of less than 0.05 was considered statistically significant.

The binary logistic regression was used to assess the relationship between the presence of PTA and outcome variables. Omnibus test of model coefficients and Hosmer and Lemeshow test were used for testing model. Cox \& Snell R square and Nagelkerke R square were also tested. The Wald test was used for the significant variables in the model.

\section{RESULTS}

Two-hundred and seventy-two patients underwent kidney transplantation during 2014 to 2015. Thirteen patients died or lost graft within one year were excluded. Therefore, 259 patients were included and investigated in our study. Most patients who developed anemia at 1 year were female $(P=0.01)$. The CIT was significantly longer in anemia group than that in no anemia group $(11.5 \pm 9.6$ hours and $8.2 \pm 9.8$ hours, respectively, $P=0.05$ ). As for induction therapy, the use of ATG appeared to be higher in anemia group $(23 \%, P=0.03)$ whereas no induction therapy seemed to occur more in no anemia group (33.6\%, $P=0.03)$. We found a higher number of ECD $(23.9 \%, P=0.01)$ and a lower number of living donor $(38.1 \%, P=0.01)$ in anemia group when compared with those donor factors in no anemia group, respectively. Baseline characteristics of kidney transplant patients categorized by anemia incidence at oneyear follow-up period were shown in Table 1.

\section{The incidence of PTA during one year transplantation}

At the immediate phase $(<14$ days after kidney transplantation), the incidence of PTA was in the range of 87 to $95 \%$. The number of patients with PTA gradually decreased overtime. There were $58.8 \%$ and $43.6 \%$ of kidney transplant patients having anemia at month 3 (early phase), and 12 (late phase), respectively (Figure 1 and 2). At 12 months after kidney transplantation, $\mathrm{Hb}$ level was $11.3 \pm 1.3 \mathrm{~g} / \mathrm{dL}$ and $14.6 \pm 1.6 \mathrm{~g} / \mathrm{dL}$ in 


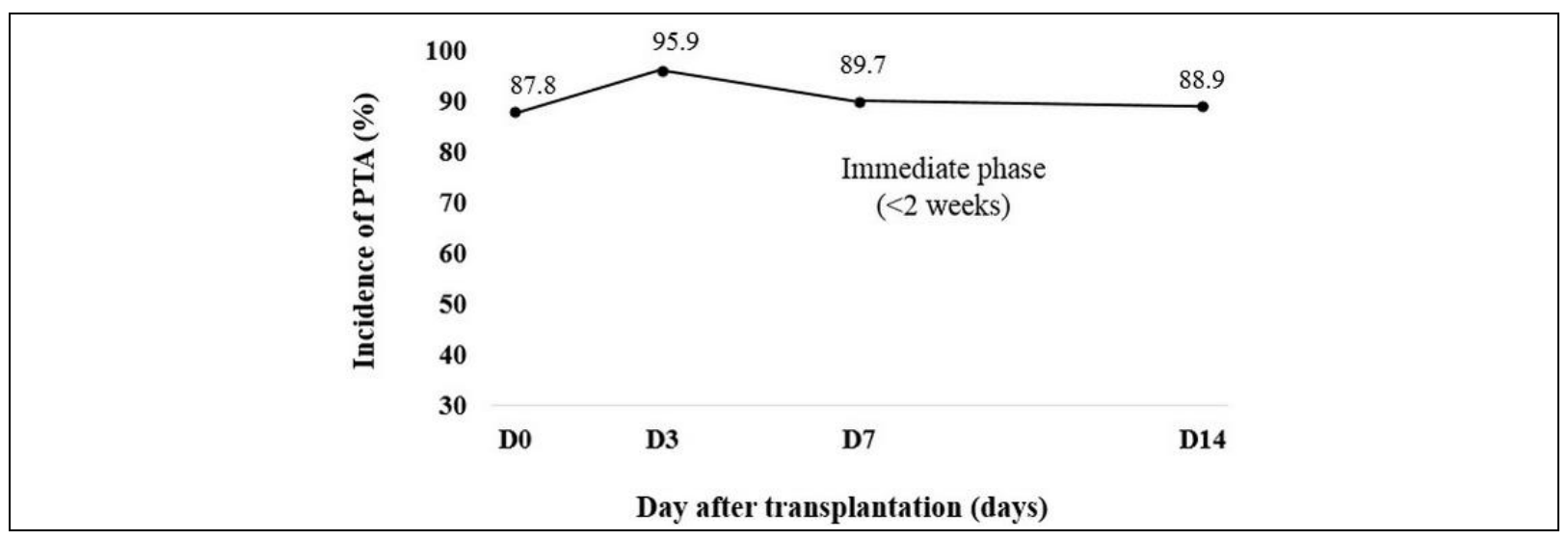

Figure 1. The incidence of PTA during the immediate phase ( $<2$ weeks) after transplantation

anemia and no anemia group, respectively $(P<$ $0.001)$. The values of eGFR at one year were $56.7 \pm 20.6 \mathrm{~mL} / \mathrm{min} / 1.73 \mathrm{~m}^{2}$ and $62.8 \pm 20.2$ $\mathrm{mL} / \mathrm{min} / 1.73 \mathrm{~m}^{2}$ in anemia and no anemia group, respectively $(P=0.017)$.

Medication- related anemia including ganciclovir, valganciclovir, ACEI/ARB, folic acid supplement, iron supplement, vitamin B6 complex supplement were not different between anemia and no anemia group. UTI was the most common infection in our studied patients. The incidence of UTI was $65 \%$ and $44.5 \%$ in anemia and no anemia group, respectively $(P=0.001)$. Sepsis, viremia and viruria were not commonly found and not different between anemia and no anemia group.

\section{Factors associated PTA}

Binary logistic regression (binomial regression model) was used to select variables to create best association and prediction of PTA, the 11 independent variables shown in Table 2 were among the factors that offered best prediction from this analysis. Factors associated PTA which were divided into the increased risk and decreased risk of PTA. Factors associated increased risk of PTA including UTI, ganciclovir or valganciclovir, history of using rHuEpo, duration of ESRD, and recipient's age. Factors associated decreased risk of PTA included duration after transplantation, eGFR after transplantation, $\mathrm{Hb}$ at baseline, standard criteria donor, and living donor. The relationship between variables and PTA incidence was provided in Table 2. As model evaluation, the predicted probability of PTA equation was

$\log (\mathrm{P}) /(1-\mathrm{P})=0.775(\mathrm{~A})+0.420(\mathrm{~B})+$ $0.394(\mathrm{C})+0.323(\mathrm{D})+0.032(\mathrm{E})+0.019(\mathrm{~F})-$ $0.006(\mathrm{G})-0.011(\mathrm{H})-0.187(\mathrm{I})-1.064(\mathrm{~J})-$ $1.237(\mathrm{~K})+3.839$

When $\mathrm{P}=$ Probability of PTA, $1-\mathrm{P}=$ Probability of no PTA, A = urinary tract infection, $\mathrm{B}=$ ganciclovir or valganciclovir, $\mathrm{C}=$ history of blood transfusion, $\mathrm{D}=$ history of using $\mathrm{rHuEpo}, \mathrm{E}=$ duration of $\mathrm{ESRD}, \mathrm{F}=$ recipient' $\mathrm{s}$ age, $\mathrm{G}=$ duration after transplantation, $\mathrm{H}=\mathrm{eGFR}$ after transplantation, $\mathrm{I}=\mathrm{Hb}$ at baseline, $\mathrm{J}=$ standard criteria donor, $\mathrm{K}=$ living donor

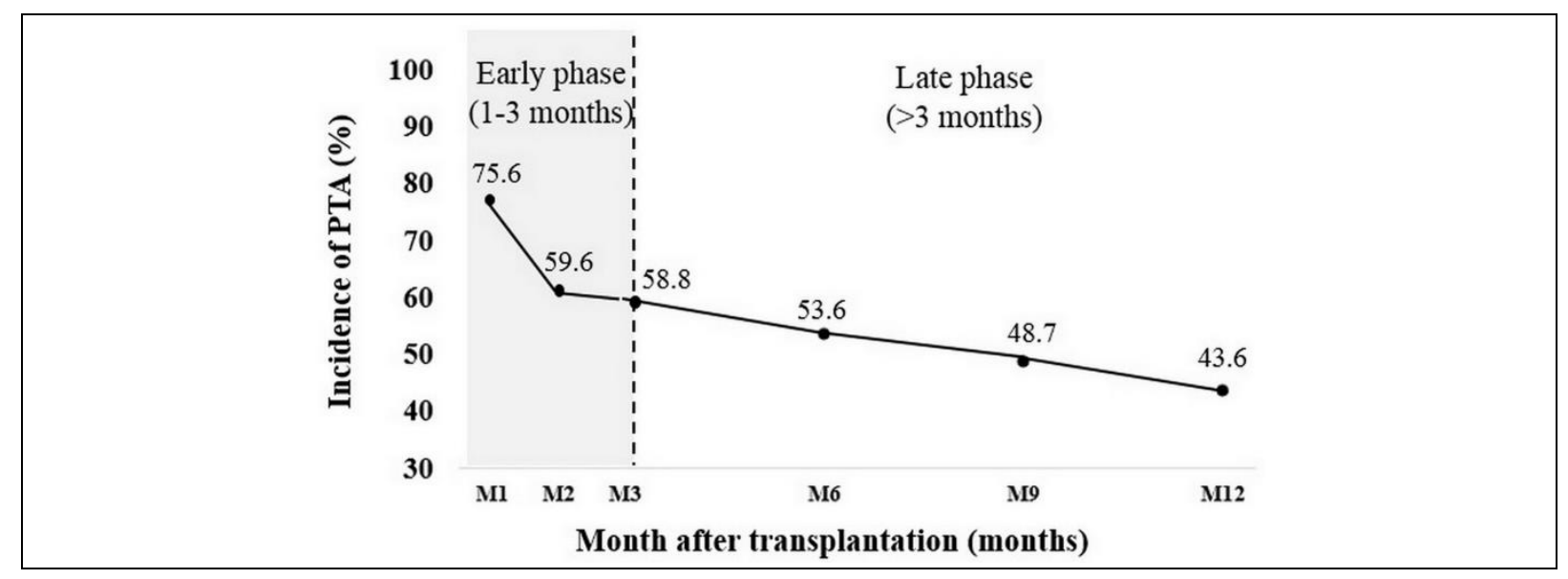

Figure 2. The incidence of PTA during the early phase (1-3 months) and late phase ( $>3$ months) after transplantation 
Table 2 The relationship between variables and PTA incidence (Binary regression analysis)

\begin{tabular}{clccc}
\hline & \multicolumn{1}{c}{ Variables } & Odds & $\mathbf{9 5 \%}$ CI & $\boldsymbol{P}$-value \\
\hline A & Urinary tract infection & 2.170 & $1.434-3.248$ & $0.001^{*}$ \\
B & Ganciclovir or valganciclovir & 1.522 & $0.926-2.501$ & 0.097 \\
C & History of blood transfusion & 1.483 & $1.177-1.868$ & $0.001^{*}$ \\
D & History of using rHuEpo & 1.381 & $1.098-1.737$ & $0.006^{*}$ \\
E & Duration of ESRD & 1.032 & $1.006-1.059$ & $0.015^{*}$ \\
F & Recipient's age & 1.019 & $1.009-1.030$ & $0.001^{*}$ \\
G & Duration after transplantation & 0.994 & $0.993-0.995$ & $0.001^{*}$ \\
H & eGFR after transplantation & 0.989 & $0.985-0.994$ & $0.001^{*}$ \\
I & Hb at baseline & 0.829 & $0.776-0.886$ & $0.001^{*}$ \\
J & Standard criteria donor & 0.345 & $0.220-0.542$ & $0.001^{*}$ \\
K & Living donor & 0.290 & $0.186-0.453$ & $0.001^{*}$ \\
\hline
\end{tabular}

eGFR = estimated glomerular filtration rate, $\mathrm{ESRD}=$ end stage renal disease, $\mathrm{Hb}=$ hemoglobin, $\mathrm{rHuEpo}=$ recombinant human erythropoietin; ${ }^{*} P$-value of less than 0.05 was considered statistically significant

This equation had an accuracy of predicted percentage of anemia of 75.3

\section{DISCUSSION}

Our study in Thai kidney transplant patients showed the incidence of PTA varying in different period of time after transplantation. Almost all patients $(>85 \%)$ had anemia during immediate phase $(<2$ weeks $)$ whereas a notable decrease in PTA incidence was found during early phase (1-3 months). As the late phase (3 months to 1 year), PTA incidence tended to be consistent with approximately $40 \%$ of all kidney transplant patients. Many factors were associated with increased risk and decreased risk of PTA with a high percentage of anemia prediction.

Finding pertinent factors are of importance in the aspect of early recognition and improvement of modifiable factors associated PTA. Our patient characteristics revealed that most of patients having anemia were female which may reflect the contribution of menstruation in premenopausal women ${ }^{11}$. Renal function after transplantation has a great impact on EPO synthesis leading to the resolution of anemia ${ }^{1-2,8}$. As organ transplantation has a unique characteristics affecting graft function, a good quality of kidney allograft has a positive influence on renal function after transplantation ${ }^{12}$. We found that deceased donor with high CIT or ECD, which generally tends to have a poor graft function, was higher in patients having anemia. Similarly, living donor, which commonly provides a good graft function, was lower in patients having anemia. Furthermore, medication used as induction therapy can be a predictor of renal graft function. In general, no induction has been considered for patients having standard risk of rejection whereas ATG has been prescribed for patients having high risk of rejection ${ }^{13}$. The induction therapy supported the PTA incidence of which the former significantly decreased risk and the latter significantly increased risk as found in our kidney transplant patients.

We can imply a high percentage of predicted probability of anemia in that the risk of anemia will be diminished if all specific factors are managed properly. Practitioners can recognize the tendency of anemia since before transplantation as regards unmodifiable factors found in the study. Patients having history of blood transfusion before kidney operation tended to have inherit factors for EPO hyporesponsiveness such as hemoglobinopathies, bone marrow failure, EPO resistance which could interfere the resolution of anemia after transplantation ${ }^{2}$. Patients having rHuEpo used before transplantation also showed an increase in PTA incidence, which was consistent with the finding from Kitamura $\mathrm{K}$, et $\mathrm{al}^{14}$. Hb level at baseline (before kidney operation) also proved that recipients' anemia status before transplantation has an impact on PTA. In addition, older patients as well as a longer duration of ESRD contributed to be more risk of graft failure. The history of prolonged maintenance dialysis affected systemic immune response leading to inflammation process which can carry over a low EPO response after kidney transplantation $^{1,14}$. Donor factors related good graft function (standard criteria donor and living donor) were also found to be decreased risk of PTA.

A higher eGFR and a longer time after transplantation showed a positive impact for the resolution of anemia. The values of eGFR at one year also supported that patients not having anemia were significantly higher eGFR 
compared with that in patients having anemia. We should be aware of whether we allow an adequate time for fully renal recovery along with correcting other causes affecting renal function before approaching anemia complication. It would be interesting that infection, specifically UTI, was associated with increased risk of PTA. UTI has resulted in deterioration of renal function as inflammatory markers (IL- 6, IL- 8) were elevated leading to kidney allograft inflammation ${ }^{15}$. Ganciclovir/valganciclovir was found to be associated with PTA as they cause inherited bone marrow suppression ${ }^{16}$. From our point of view, anemia will be resolved when specific factors are corrected (UTI treatment/discontinuation of the causative agents). We did not find other common drugs causing anemia in our study. As all transplant patients normally received mycophenolic acid (MPA) as immunosuppressant, however, the relationship between MPA and PTA incidence was not found. Moreover, a limited number of patients who were prescribed sirolimus, azathioprine and ACEI/ARBs during one year after transplantation in our center had led to no association with PTA. Although CNIs (tacrolimus, cyclosporine) and TMP/SMX, for (Pneumocystis carrinii pneumonia; PJP) prophylaxis, could cause anemia problem ${ }^{16}$, we routinely provide these medications for all kidney transplant patients which were not presented as factors for PTA incidence.

As a nature of a retrospective study, certain laboratory parameters related anemia such as iron status, infection, AKI incidence, biopsy proven or suspected acute rejection were loss or incomplete record. Studies evaluating the optimal $\mathrm{Hb}$ target and the role of rHuEpo for correcting anemia in transplant patients will be further required. Long term follow-up study will provide additional detail toward the current knowledge of PTA.

\section{CONCLUSIONS}

Post-transplantation anemia (PTA) is the common complication in Thai kidney transplant patients as found $43.6 \%$ at one year after transplantation. We proposed the predicted probability of PTA both factors increased risk and decreased risk of anemia. Early recognition by finding of the anemic tendency along with management all possible causes helpfully provides the better care of anemia complication for kidney transplant patients.

\section{ACKNOWLEDGEMENTS}

Part of this study was presented at the US- Thai consortium meeting, College of Pharmacy, University of Kentucky, Lexington, KY, 10-12 June, 2018. All authors would like to thank Jiraporn Pramongmook, Thanaporn Sangdet for data collection and a part of data analysis.

Conflict of interest (If any)

All authors have no conflict of interest to declare.

\section{Funding}

None to declare

\section{Ethical approval}

The study was approved by the Ethical Clearance Committee on Human Rights Related to Research Involving Human Subjects, Faculty of Medicine, Ramathibodi Hospital, Mahidol University (MURA 2017/606)

\section{Article info:}

Received April 16, 2019

Received in revised form October 4, 2019

Accepted February 12, 2020

\section{REFERENCES}

1. Winkelmayer WC, Chandraker A. Posttransplantation anemia: management and rationale. Clin $\mathrm{J}$ Am Soc Nephrol. 2008;3(Suppl 2):S49-55.

2. Kidney Disease: Improving Global Outcomes (KDIGO). KDIGO 2017 Clinical practice guideline for anemia in chronic kidney disease. Kidney Int (Suppl). 2012;2:279-335

3. The Renal Association. Clinical practice guideline anaemia of chronic kidney disease [ document on the internet]. 2017 [updated 2017 June; cited 2019 October 2]. Available from: https://renal.org/guidelines/.

4. KDOQI Clinical practice guidelines and clinical practice recommendations for anemia in chronic kidney disease. Am J Kidney Dis. 2006;47(5 Suppl 3):S11-145.

5. WHO Scientific Group on Nutritional Anaemias \& World Health Organization. Nutritional anaemias: report of a WHO scientific group [ meeting held in Geneva from 13 to 17 March 1967] [document on the internet] . World Health Organization; 1968 [cited 2019 October 2] . Available from: https://apps.who.int/iris/ handle/10665/40707.

6. Charan J, Biswas T. How to calculate sample size for different study designs in medical research? Indian J Psychol Med. 2013;35(2):121-6.

7. Imoagene-Oyedeji AE, Rosas SE, Doyle AM, Goral S, Bloom RD. Posttransplantation anemia at 12 months in kidney recipients treated with mycophenolate mofetil: risk factors and implications for mortality. J Am Soc Nephro. 2006;17(11):3240-7.

8. Bamgbola OF. Spectrum of anemia after kidney transplantation: pathophysiology and therapeutic implications. Clin Transplant. 2016;30(10):1185-94.

9. Chhabra D, Grafals M, Skaro AI, Parker M, Gallon L. 
Impact of anemia after renal transplantation on patient and graft survival and on rate of acute rejection. Clin J Am Soc Nephrol. 2008;3(4):1168-74.

10. Chang Y, Shah T, Min DI, Yang JW. Clinical risk factors associated with the post-transplant anemia in kidney transplant patients. Transpl Immunol. 2016;38:50-3.

11. Blosser CD, Bloom RD. Posttransplant anemia in solid organ recipients. Transplant Rev (Orlando). 2010;24(2):89-98.

12. Wagner SJ, Brennan DC. Induction therapy in renal transplant recipients: how convincing is the current evidence? Drugs. 2012;72(5):671-83.

13. Thai Transplantation Society. Thai Transplant Care
14. (TTC): Kidney [document on the internet]. Thailand; 2014 [updated 2014 September; cited 2019 October 2]. Available from: http://transplantthai.org.

15. Kitamura K, Nakai K, Fujii H, Ishimura T, Fujisawa M, Nishi S. Pre-transplant erythropoiesis-stimulating agent hypo- responsiveness and post- transplant anemia. Transplant Proc. 2015;47(6):1820-4.

16. Ciszek M, Paczek L, Bartlomiejczyk I, Mucha K. Urine cytokines profile in renal transplant patients with asymptomatic bacteriuria. Transplantation. 2006;81(12):1653-7.

17. Yabu JM, Winkelmayer WC. Posttransplantation anemia: mechanisms and management. Clin J Am Soc Nephrol. 2011;6(7):1794-801. 